\title{
Tronchet et la réorganisation constituante des institutions judiciaires
}

Tronchet and the Reorganization of Judicial Institutions during the Constituent Assembly

\section{Philippe Tessier}

\section{(2) OpenEdition}

Journals

\section{Édition électronique}

URL : https://journals.openedition.org/ahrf/11200

DOI : $10.4000 / a h r f .11200$

ISSN : 1952-403X

Éditeur :

Armand Colin, Société des études robespierristes

Édition imprimée

Date de publication : 1 décembre 2007

Pagination : 9-26

ISSN : 0003-4436

Référence électronique

Philippe Tessier, «Tronchet et la réorganisation constituante des institutions judiciaires », Annales historiques de la Révolution française [En ligne], 350 | octobre-décembre 2007, mis en ligne le 01 janvier 2011, consulté le 01 juillet 2021. URL : http://journals.openedition.org/ahrf/11200 ; DOI : https:// doi.org/10.4000/ahrf.11200 
$\begin{array}{lllllllll}\text { A } & R & \mathbf{T} & \text { I } & \mathbf{C} & \mathrm{L} & \mathrm{E} & \mathrm{S}\end{array}$

\title{
TRONCHET ET LA RÉORGANISATION CONSTITUANTE DES INSTITUTIONS JUDICIAIRES
}

\author{
Philippe TESSIER
}

\begin{abstract}
François Denis Tronchet, connu pour sa participation au procès du roi et sa contribution à la rédaction du Code civil de 1804, joua un rôle important, pendant la Constituante, dans la réorganisation des institutions judiciaires d'Ancien Régime, et, notamment, dans la réforme de la procédure criminelle. Bien que très tôt qualifié de "Nestor de l'aristocratie ", il apparaît à la fois comme attaché à certaines traditions et ardent promoteur de la réforme, aussi peutil être qualifié d'homme de la transaction. En fait, Tronchet utilise l'expérience qu'il a acquise dans sa pratique du métier d'avocat pour élaborer des solutions pragmatiques, concrètes et humaines. Ces solutions de compromis sont souvent inspirées par un idéal de modération, de défense de l'équilibre et du refus de la "barbarie ", ce concept repoussoir qui désigne à la fois l'Ancien Régime et certaines pratiques étrangères ; cet ensemble d'idées est étroitement associé au modèle de l'avocat, qu'il glorifie, tout en rejetant le corporatisme judiciaire.
\end{abstract}

Mots-clés : droit criminel, réforme de la justice, transaction, barbarie, avocat.

Les polémiques autour des " causes célèbres "', les scandales des affaires Calas, Sirven et La Barre, et les débats sur la réforme pénale avaient mis les questions de justice au cour des préoccupations des hommes de la fin du XVIII siècle. Lanalyse des cahiers de doléances effectuée par Gilbert Shapiro et John Markoff démontre que les revendications concernant l'organisation du pouvoir politique, et notamment la justice,

(1) Sarah MAZA, Vies privées, affaires publiques, les causes célébres de la France prérévolutionnaire, Paris, Fayard, 1997. 
sont plus fréquentes que les demandes économiques et sociales ${ }^{2}$. Les cahiers dénoncent la complexité de la justice, son caractère inégalitaire, sa partialité, l'absence de garanties fournies à la liberté individuelle, le secret de la procédure et la vénalité des offices ${ }^{3}$. En 1789, c'est ainsi une réforme attendue des institutions judiciaires et des procédures qu'entame l'assemblée.

Les enjeux - politiques et juridiques - sont majeurs : il s'agit de garantir à la fois la liberté individuelle et la sûreté publique et, au travers d'une réorganisation globale des institutions judiciaires, de mettre la magistrature à l'écart du pouvoir politique, de la soumettre à la souveraineté nationale et, à certains égards, de la confiner dans un rôle d'exécution. Les débats aboutissent au décret des 16-29 septembre 1791, qui abroge l'ordonnance de 1670 et réorganise la procédure criminellet. Entre temps, le décret des 8-9 octobre 1789 réforme provisoirement cette même procédure en instaurant le principe de la publicité et en assurant l'assistance de l'accusé par un conseils.

Ces travaux de la Constituante sont animés, au premier chef, par des hommes de loi, anciens avocats ou magistrats, nombreux en son sein". En ce domaine, la part de François-Denis Tronchet est rarement évoquée. Son rôle dans l'élaboration du Code civil de 1804', pourtant souvent éclipsé par celui de Portalis ${ }^{x}$, a peut-être contribué à minimiser son intervention dans la réforme des institutions judiciaires de l'Assemblée constituante. Pourtant, à revenir sur la participation de Tronchet aux débats de l'Assemblée, comment ne pas souligner que ses positions, pas toujours bien comprises $^{9}$, furent étonnamment clairvoyantes, et parfois déterminantes?

(2) Gilbert Shapiro, John Markoff, Revolutionary Demands, A content Analysis of the Cahiers de Doléances of 1789 , Stanford, Stanford University Press, 1998, p. 266, tableau 14-1. Les doléances concernant la justice apparaissent dans $13 \%$ des cahiers de doléances.

(3) Jean Claude FarCY, L'Histoire de la Justice en France de la Révolution à nos jours, Paris, PUF, 2001, p. 141-142.

(4) Jacqueline Lucienne LAFON, La Révolution Française face au système judiciaire d'Ancien Régime, Paris, Droz, 2001, p. 74.

(5) Ihid.

(6) Jean-Louis Halpérin, L'impossible Code civil, Paris, PUF, 1992.

(7) Patrick Weil, Qu'est-ce qu'un Français ?, Paris, Gallimard, 2002, p. 41 et suiv. Patrick Weil démontre notamment l'importance que prit Tronchet dans l'élaboration du droit de la nationalité.

(8) Alors même que Napoléon à Sainte-Hélène le qualifie " d'âme du Code civil ". LAS CASES, Mémorial de Sainte Hélène, Paris, Garnier, 1854, t. I, p. 361 : "Tronchet en était l’âme, disait Napoléon, et lui, le démonstrateur. Tronchet avait un esprit éminemment profond et juste, mais il sautait par-dessus les développements, parlait fort mal et ne savait pas se défendre ". Portalis avait une belle voix et était donc mis en valeur dans les apparitions publiques de la commission, contrairement à Tronchet, qui, bien que président, restait en retrait.

(9) Adhemar Esmein, A History of Continental Criminal Procedure, with Special Reference to France, London, J. Murray, 1914, p. 425, « la motion de Tronchet sur la procédure écrite dans le jury n'avait vraiment rien à voir avec la question $n$. 
Tronchet, dernier bâtonnier de l'ordre des avocats de Paris, élu du Tiers état de la capitale et jadis acteur de la contestation contre la réforme Maupeou ${ }^{10}$, paraît à la fois promoteur de la réforme et attaché à certaines traditions ; en cela, il peut être qualifié d'" homme de la transaction". Quel contraste, en effet, entre l'image du personnage construite à l'issue de la Constituante ou du procès du roi - celle d'un "monarchien " ou d'un "Nestor de l'aristocratie " ", et l'enthousiasme de l'homme pour la réforme du droit criminel, ou sa dénonciation des tentations dilatoires de la Constituante $^{12}$ ! Il faut dès lors nuancer la légende, formée à une époque marquée par une accélération rapide des événements qui forçait systématiquement les modérés à choisir leur camp ${ }^{\prime 3}$. Les contradictions apparentes du personnage méritent d'être mises à jour et analysées. Derrière elles, c'est sans doute le juriste, soucieux des techniques et des logiques juridiques, et faisant profiter l'Assemblée de son expertise qui transparaît ; à cet égard, Tronchet est la version révolutionnaire de "l'expert ", l'ancêtre, peut-être, du technocrate actuel. Mais il est aussi le pragmatique, qui se montre en permanence séduit par un art du compromis, et qui traduit à la fois un choix politique et une culture professionnelle qu'il continue de glorifier tout en rejetant le corporatisme judiciaire. En cela, il incarne l'idéal de l'avocat, attaché aux valeurs de l'honneur, de l'équilibre et du désintéressement.

Aux États généraux et à la Constituante, François-Denis Tronchet est loin de rester inerte ; Edna Lemay et Timothy Tackett le recensent parmi les orateurs les plus prolixes de l'Assemblée. Avec ses 196 discours, c'est le $33^{e}$ orateur en nombre d'interventions ${ }^{14}$. Il est, de plus, membre de nombreux comités, et notamment du comité de Législation criminelle (14 septembre 1789- 22 janvier 1790) $)^{15}$. Par ses interventions et son travail, il est ainsi au cœur de la réforme qui s'annonce.

(10) Lanalyse du volume IV des consultations de François-Denis Tronchet démontre qu'il ne rédigea aucune consultation du mois de décembre 1770 au mois de décembre 1771

(11) André Jean ARnaub, Les ongines doctrinales du Code civil français, Paris, Librairie générale d'histoire du droit et de jurisprudence, 1969, p. 31. Voir aussi Edmond Louis Alexis Dunols de CrancE, Le Véritable portrait de nos législateurs, ou Galerie des tableaux exposés à la vue du public depuis le 5 mai 1789

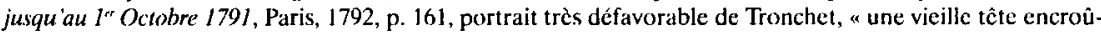
tée d'aristocratie ".

(12) Voir le rapport du 25 décembre 1789. Cf. Roberto Martucc, La Costituente ed il problema penale in Francia, 1789-1791, Milano, A. Giuffrè, 1984, p. 179.

(13) Jcan Clément Martin, Révolution, Contre-Révolution et Nation, Paris, Seuil, 1998, p. 46 et suiv.

(14) Edna H. Lemay, « Les révélations d'un dictionnaire : du nouveau sur la composition de l'assemblée nationale constituante (1789-1791) n, AHRF, n²84, 1991, p. 159-189; Timothy TAcKETT, Par la volonté du peuple, comment les députés de 1789 sont devenus Révolutionnaires, Paris, Albin Michel, 1997, annexe IJ. p. $904-906$

(15) Voir Edna H. Lfmay, Dictionnaire des Constituants, 1789-1791, Paris, Universitas, 1991, 
En matière judiciaire, les principales interventions de Tronchet s'échelonnent du 24 décembre 1789 au mois de juillet 1791. Les plus importantes portent sur les difficultés suscitées par l'application de la première réforme de la procédure criminelle des 8 et 9 octobre $1789^{11}$, sur la question de la sédentarité des tribunaux d'appel ${ }^{17}$, sur l'organisation du tribunal de cassation ${ }^{18}$, sur la question de l'introduction du jury en matière criminelle ${ }^{19}$ et sur le jury criminel de jugement et la procédure écrite ${ }^{20}$. Tronchet intervient également sur le statut à réserver à l'accusé contumax ${ }^{21}$, sur la prescription et le jugement ${ }^{22}$, sur les tutelles.

En fait, on retrouve Tronchet dès les premières mesures de l'Assemblée. Dès le 10 septembre 1789, n'est-il pas élu membre du Comité de Législation criminelle, également appelé Comité des Sept chargé de quelques réformes du droit criminel $^{23}$ et, à ce titre n'est-il pas associé à l'élaboration du décret des 8 et 9 octobre 1789, dit " décret Beaumetz ", qui proroge les dispositions non modifiées de l'ordonnance de 1670, tout en instaurant trois nouveautés : la publicité de la procédure après le décret d'accusation, la possibilité pour l'accusé de prendre un conseil, et de faire admettre ses justifications ${ }^{2+}$ ? Le décret des 8 et 9 octobre 1789 aboutit aussi à la naissance d'une institution promise à une courte existence, celle des notables-adjoints, qui doivent surveiller l'instruction. Si le décret Beaumetz se veut provisoire, il n'en instaure pas moins une obligation de publicité qui bouleverse profondément la procédure criminelle, dès sa promulgation par le roi le 12 octobre 1789.

En effet, la révision des 8-9 octobre 1789 redéfinit l'organisation du procès autour d'un décret rendu par le magistrat, qui rend la procédure contradictoire avec l'accusé ; auparavant, la procédure restait secrète du début à la fin, les droits de l'accusé demeurant limités et sa possibilité de constituer avocat pratiquement impossible au grand criminel. La procédure nouvelle se divise donc en deux phases : une première phase « de publicité atténuée ${ }^{25}$, avant le décret du magistrat ; une seconde phase qui est soumise à publicité intégrale. Pendant la phase de publicité atténuée, l'ins-

(16) Archives Parlementaires, t. XI, p. 3, séance du 24 décembre 1789, rapport de Tronchet sur le mémoire de M. Le Garde des Sceaux concernant les difficultés qui se sont élevées pour l'exécution du décret des 8 et 9 octobre dernjer sur la procédurc criminelle.

(17) Archives parlementaires, 2 mai $1790, t . X V$, p. 361 et suiv.

(18) Ibid., 25 mai 1790 , t. XV, p. 671 et suiv.

(19) Ihid., 5 juillet 1790 , t. XVI, p. 705.

(20) Ibid., 5 janvier 1791 , t. XXII, p. 27 et suiv. Voir aussi séances des 18 et 19 janvier 1791.

(21) Ibid., I" février 1791, t. XXII, p. 661 et suiv.

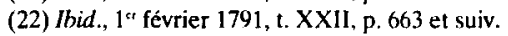

(23) Laurent Drugeon, La réforme de la justice pénale avant la mise en place du jury. Thèse d'histoire du droit soutenue le 10 décembre 2004, sous la direction d'André Laingui, Paris II Panthéon Assas, t. 1, p. 185 et suiv.

(24) Ihid., p. 194

(25) Jacqueline Lucienne LAFON, op. cit., p. 75. 
titution des notables-adjoints - des citoyens désignés par la municipalité pour surveiller l'action du juge - est un moyen de compenser le désavantage qui résulte du secret pour l'accusé. On voit ici que la publicité est un élément fondamental de la réforme. Tronchet, en décembre 1789, l'expose ainsi : "La publicité de la procédure criminelle, voilà le principal point de réformation provisoire que vous avez désiré, et que vous avez voulu opérer par votre décret des 8 et 9 octobre $"$ "20.

À cet égard, une des principales interventions de Tronchet à la Constituante est un Rapport sur le mémoire de M. le Garde des Sceaux concernant les difficultés qui se sont élevées pour l'exécution du décret des 8 et 9 octobre dernier sur la procédure criminelle" ${ }^{27}$. Le Garde des Sceaux, Champion de Cicé, envoie en effet au président de l'Assemblée nationale, le 8 décembre 1789, un mémoire sur les difficultés d'application résultant du décret des 8 et 9 octobre 1789, et notamment sur l'institution des notables-adjoints. Confronté à ce texte novateur, le personnel judiciaire s'est trouvé ponctuellement désemparé, comme en témoigne la correspondance que les différents tribunaux entretiennent avec Champion de Cicé $^{28}$ ou les lettres et les adresses envoyées au Comité de Judicature ${ }^{29}$. Les difficultés d'application proviennent de la réticence des magistrats à collaborer avec des notables qui ne connaissent pas bien le droit, et dont l'origine sociale est souvent différente de la leur, mais aussi de la réticence des notablesadjoints à assumer leur mission ${ }^{30}$. Le rapport de Tronchet est une réponse au mémoire du Garde des Sceaux ${ }^{31}$. Le député y apparaît partisan de la réforme de l'ordonnance de 1670 , mais déplore l'absence d'une réorganisation plus globale et plus immédiate de la procédure criminelle. Alors que, au nom du Comité des Sept, en octobre 1789, Beaumetz avait évoqué la nécessité d'une « sage lenteur » dans la réorganisation de la justice, et que le comité avait disposé que toutes les institutions judiciaires d'Ancien Régime seraient provisoirement prorogées, Tronchet semble désirer une réforme plus complète : "L'Assemblée nationale a réservé pour un autre moment de délibérer sur l'instruction des jurés, plan plus vaste, qui exigerait une réformation totale de nos lois criminelles, non seulement sur la forme de la procédure, mais encore sur toutes nos lois pénales; elle n'a voulu admettre qu'une réformation provisoire, qui pût admettre une publicité, en se conciliant avec les institutions anciennes et les usages connus. Son comité a dû se renfermer dans des pouvoirs limités ${ }^{32} \ldots$ Il y a ici une

(26) Archives parlementaires, t. IX, p. 214.

(27) Ihid., 24 décembre 1789 , t. XI, p. 3 et suiv.

(28) AN, BB 1.58 .

(29) Je reprends ici un passage de la thc̀se de Laurent Drugeon, op. cit., p. 205.

(30) Jacquetine Lucienne LAFON, op.cit., p. 78-79.

(31) Archives parlementaires, t. XI, p. 3-12.

(32) Ibid., t. XI, p. 5. 
déploration discrète, qui demande une refonte plus vaste de la procédure criminelle, et refuse son caractère provisoire, motivé en partie par la crainte des désordres populaires et les difficultés d'application par les magistrats.

Lors de la séance du 5 janvier 1791, et lors des débats sur le jury de jugement et la procédure écrite, Tronchet propose une refonte plus complète de l'ordonnance de 1670 : « Nous sommes bien éloignés de réclamer l'ancienne forme de procédure, que vous aviez déjà réformée par votre décret provisoire ${ }^{33}$, et que je proposerai de réformer d'une manière encore plus complète, puisque j'entends que toute l'instruction se fasse publiquement et en présence des jurés ${ }^{34}$. Tronchet ne dissimule pas son enthousiasme pour les premiers changements révolutionnaires apportés à la procédure criminelle : «L'humanité réclamait depuis longtemps en faveur de l'accusé une procédure qui, déchirant le voile qui cachait à l'accusé la marche de l'accusateur, pût le mettre à portée de combattre à armes égales avec l'accusateur et le juge $»^{35}$. Tronchet affirme ici une inclination surprenante, pour un juriste dépeint comme " positiviste $»^{36}$, envers la procédure accusatoire, à l'anglaise, où la défense et l'accusation sont des parties égales en droit devant le juge qui est l'arbitre de leur combat, par opposition à la procédure inquisitoire de l'ordonnance de 1670 , où le juge peut instruire à charge et à décharge sans que l'accusé puisse prendre de réelle initiative procédurale. Comme il le réaffirme plus bas, "la publicité de l'instruction et du jugement est le frein le plus puissant que la loi puisse opposer aux erreurs, à la faiblesse, à la négligence ou à la corruption du juge, à l'indiscrétion ou à la corruption des témoins $~^{37}$. Pour Roberto Martucci, «l'exigence de rendre public le procès pénal avait présidé à la réforme d'octobre qui avait poursuivi le but de protéger l'intérêt général de la société sans réduire outre mesure l'intérêt particulier des accusés [...]. La majorité pensait que cette alliance d'intérêts par essence antinomiques pouvait se réaliser en adoptant la formule procédurale des anglo-saxons; dans le rapport du comité l'option accusatoire est définie avec emphase, même conçue comme un objectif non immédiatement réalisable " ${ }^{34}$. Il n'y a ici aucune trace de corporatisme judiciaire chez Tronchet, mais, bien au

(33) Référence ici au décret des 8 et 9 octobre 1789.

(34) Archives parlementaires, 5 janvier 1791, t. XXII, p. 27 et suiv.

(35) Ibid., t. XI, p. 3.

(36) André Jean ARnaud, op.cit, p. 57 : « Si nous savons peu de choses sur Tronchet, d'autant plus solide dans ses raisonnements qu'il s'en tient, positiviste résolu, aux seules dispositions des coutumes et des ordonnances, nous suivons par contre avec grand interêt les interventions de Treilhard et de Berlier ". Ici, loin de s'en tenir aux seules coutumes et ordonnances, Tronchet semble bien adhérer aux grands principes de la réforme du droit pénal.

(37) Archives parlementaires, t. XI, p. 5

(38) Roberto MARTUCCI, op. cit., chapitre V, p. 174. 
contraire, le désir, partagé par les éléments les plus avancés de la Constituante, d'équilibrer les armes des parties.

Le 5 juillet 1790, Tronchet démontre encore une fois ses aspirations au changement, en participant à la discussion sur l'instauration des jurés en matière criminelle, à propos de laquelle il propose un amendement ${ }^{39}$. Il propose également un article ainsi rédigé : «Tout privilège en matière de juridiction est aboli ; tous les citoyens, sans distinction, plaideront en la même forme, et devant les mêmes juges, dans les mêmes cas ». Cet article, qui signe la disparition des privilèges de juridiction de l'Ancien Régime, notamment du privilège de committimus, ne semble pas corroborer la réputation de Tronchet comme « Nestor de l'aristocratie ». Les aspirations réformatrices de Tronchet ne vont pas toutefois jusqu'à adhérer au principe du jury civil, qu'il refuse, avec Thouret, en soutenant que d'irréductibles différences procédurales entre la France et l'Angleterre rendent intransportables sur le continent un système de preuves de conception totalement différente où le témoignage l'emporte sur l'écrit ${ }^{\text {th }}$.

Autre manifestation de son esprit réformateur, Tronchet affirme sa méfiance vis-à-vis des parlements lors de la séance du 5 mai 1790, dans une discussion sur l'organisation judiciaire, sur la question de savoir si les tribunaux d'appel seront sédentaires ou itinérants : "Voici à quoi se réduisent toutes les objections : si vous faites des tribunaux souverains sédentaires, ce seront des parlements, et vous n'en voulez pas. Je n'en veux pas plus que vous $»^{41}$. Il affirme ici son refus des corporatismes judiciaires et la doctrine commune à la majorité des membres de la Constituante, selon laquelle les tribunaux ne sauraient s'ériger en nouveaux parlements et empiéter sur les prérogatives du pouvoir législatif et réglementaire ${ }^{42}$. Quelques semaines encore et, le 20 juillet 1790, Tronchet expose le principe qu'il faut rapprocher la justice des justiciables, et il énonce : «Non seulement il faut ouvrir aux justiciables un accès facile aux tribunaux, mais encore il faut leur procurer la meilleure justice possible $\gg^{43}$.

Ainsi, on voit que Tronchet adhère pleinement aux grands principes de la réorganisation de la justice : publicité, présence du jury, proximité, séparation par rapport au pouvoir politique. Ses positions ne sont pas simplement des avis d'expert, mais elles sont également mues par une sincère volonté de changement ; Tronchet se comporte, à l'Assemblée

(39) Archives purlementaires, 5 juiltet 1790, t. XVI, p. 705. Tronchet propose la rédaction d'un article rédigé en ces termes : "La procédure par jurés aura lieu en matière criminelle ; l'instruction sera faite publiquement et aura la publicité qui sera déterminée".

(40) Jean Pierre Royer, Histoire de la Justice en France, Paris, PUF, 1995, p. 289 et suiv.

(41) Archives partementaires, 2 mai 1790, t. XV. p. 361 et suiv.

(42) Préeccupation qui mena à l'adoption de la loi des 16-24 août 1790, qui interdisait aux juges de rendre des arrêts de règlement et de trancher par voie générale et règlementaire. Voir Jean-Pjerre ROYER, op.cit., p. 263 et suiv.

(43) Archives parlementaires, 20 juillet 1790, t. XVI, p. 7015 et suiv. 
constituante, tantôt comme un expert, tantôt comme un homme politique, cherchant à faire triompher ses idées, tant par l'expertise du praticien que par la force du verbe.

Tronchet n'est d'ailleurs pas nécessairement un mauvais orateur, contrairement à ce que les propos de Mirabeau pouvaient laisser penser : celui-ci avait en effet déclaré, lorsqu'il présidait la séance du $l^{\text {er }}$ février 1791, et réclamant le silence pour Tronchet, que celui-ci n'avait "pas autant de voix que des lumières $\gg^{44}$. Au moins une fois, lors de la séance du 5 janvier 1791, Tronchet semble avoir fait montre d'un certain talent oratoire. À la fin de son discours sur la nécessité d'une procédure écrite lors de l'instruction devant le jury, un membre de l'assemblée demande l'impression du discours de Tronchet et du projet d'article dont il a donné lecture. Cette motion est adoptée, et Fréteau intervient alors pour demander l'ajournement de la discussion : «Puisque le discours de M. Tronchet a fait tant d'impression sur l'assemblée, il faut attendre pour juger qu'on ait eu le temps de le connaitre $»^{45}$.

Si Tronchet manifeste une indéniable volonté réformatrice, toute la valeur de sa contribution à la réflexion de la Constituante sur la réforme des institutions judiciaires tient à son expérience de praticien, qui transparait tant dans ses interventions parlementaires que dans ses consultations manuscrites, conservées aujourd'hui à la bibliothèque de la Cour de cassation $^{\text {th }}$. Cette expérience même l'amène à des solutions de compromis, non par positionnement politique, mais par désir de trouver la justice dans la juste mesure et le refus des extrêmes et, si on recherche les spécificités de sa position, on peut peut-être les trouver dans la tradition humaniste aristotélicienne ${ }^{47}$. Lidée de juste mesure et de proportionnalité semble être une des clés de lecture de la pensée de Tronchet. En effet, bien loin d'être une norme rigide assise sur des canons immuables, la proportionnalité est intrinsèquement gouvernée par la contingence, puisqu'elle se situe en rapport avec les extrêmes différents qu'on peut lui opposer dans des situations différentes. En même temps, l'idée de juste mesure et de proportionnalité se situe toujours dans une dialectique de dépassement de la volonté exprimée par les participants et de fidélité au rapport de droit, prenant alors une couleur plus factuelle et objective.

Cette pensée de la juste mesure explique sa capacité à toujours se situer en opposition aux extrêmes, jusqu'en 1804, ce qui le différencie d'autres juristes comme Merlin de Douai ; ce dernier bascula, sous la Convention, dans une conception du droit plus nettement influencée par

(44) Cité par Edna H. LEMAY, op.cit., p. 404-4015.

(45) Archives parlementaires, 5 janvier 1791, t. XXII, p. 27 et suiv.

(46) Cour de cassation, fonds Tronchet, MS 266-287.

(47) Aristote, Éthique à Nicomaque, V, 1129-1131. 
des impératifs politiques ${ }^{4 *}$. Lidée de juste mesure et de proportionnalité se manifeste dans sa position équilibrée sur la question de l'indemnisation des droits féodaux", qui lui est d'ailleurs violemment reprochée par Dubois de Crancé, dans les Véritables portraits de nos législateurs : «Tronchet porta au comité féodal une vicille tête encroûtée d'aristocratie et de la légitimité de ces droits bizarres en faveur desquels il avait plaidé toute sa vie $\aleph^{\text {sil. }}$. Ce jugement est d'autant plus dur que Tronchet prend sur les droits féodaux une position pas si éloignée de celle de Merlin, qui est, lui, couvert d'éloges par le même auteur. De fait, Merlin avait présidé la sous-commission du comité de Féodalité chargée de tracer la démarcation entre droits rachetables et droits supprimés sans indemnité, ce qui lui donnait un rôle moteur dans la suppression de ces droits, alors que Tronchet présidait la souscommission chargée de fixer les conditions du rachat, ce qui lui donnait d'entrée de jeu un rôle limitatif par rapport à cette abolition.

La capacité de Tronchet à résoudre les problèmes par ce qu'on pourrait appeler une « théorie de la pratique ", apparaît notamment au cours de la discussion sur la question des difficultés d'application posées aux magistrats par le décret Beaumetz des 8 et 9 octobre 1789 : en effet, non content de rappeler les principes inspirateurs de la réforme, il propose des solutions et un projet de décret. Il s'attache à résoudre tous les problèmes possibles en pratique et affirme que "la loi doit tout prévoir " ${ }^{5}$. Son rapport du 24 décembre 1789 est donc unc méticuleuse énumération des difficultés posées et de leur résolution possible. Pour les résoudre, il prend appui sur l'importance du principe de publicité et sur une définition révélant l'importance de la fonction des notables-adjoints : "Leur fonction est de surveiller l'instruction, d'en assurer et d'en garantir la fidélité et l'impartialité qui seules peuvent attacher la confiance publique au jugement d'absolution ou de condamnation ${ }^{52}$. Le premier problème qui se pose est celui du sort de l'accusé absent ou contumace ; aura-t-il droit lui aussi à la protection des adjoints?

Tronchet se place, pour répondre à cette question, dans la perspective de la recherche d'une juste mesure. Ainsi, il refuse l'idée que les accusés absents ou contumaces puissent être privés de toute garantie : « Nos tribu-

(48) Hervé Leuwers, Merlin de Douai, un juriste en politique, 1754-1838, Arras, Artois Presse Université, 1996.

(49) Cf. Dale VAN KI.EY, The French Idea of Freedom, The Old Regime and the Declaration of the Rights of 1789, Stanford University Press, 1994, p. 332 : " le juriste François-Denis Tronchet, tout en manifestant sa réticence à contrecarrer les intentions sous-tendant la lơ du 4-âût, reconnaissait qu'un schéma juste, rationnel et universel d'indemnisation de la propriété ne devait pas impliquer un total mépris des lois coutumières touchant à la proprićtć, et des précédents, mais une analyse précise et exhaustive de leurs avantages et de leurs désavantages ".

(50) Edmond Louis Alexis Dubors de CrancE, op. cit., p. 161.

(51) Archives parlementaires, p. 7, 24 décembre 1789.

(52) Ibid., p. 4, 24 décembre 1789. 
naux français n'ont point admis la maxime barbare de certains jurisconsultes anciens, que le contumace est présumé coupable ; un grand nombre de circonstances peuvent excuser son absence $»^{53}$. Le refus de la solution extrême s'associe ici avec la réaffirmation d'une jurisprudence traditionnelle des tribunaux d'Ancien Régime ; on voit aussi que ce dernier n'est donc pas systématiquement synonyme de "barbarie " dans l'esprit de Tronchet. Toutefois, il rejette la vieille institution de la purge de la contumace, règlementée par l'article 18 du titre XVII de l'ordonnance criminelle de 1670, qui annulait toutes les formalités procédurales déjà effectuées en les convertissant in simplicem citationem, en une simple citation à comparaître $^{54}$. Pour lui, s'il est inadmissible de penser que le procès instruit durant la contumace ne produise aucun effet, il est suffisant que l'absence de l'accusé soit quelquefois excusable pour que la loi ne puisse considérer comme indigne de protection un accusé en fuite.

En décembre 1789, Tronchet affirme donc la position du Comité de jurisprudence criminelle : l'absence ou la contumace de l'accusé décrété d'accusation ne doit pas faire rappeler les adjoints aux actes postérieurs au décret. Après le décret d'accusation, toute la procédure devient totalement publique ; il n'est donc pas nécessaire de faire revenir les adjoints ; avant le décret d'accusation, les adjoints doivent de toute façon contrôler les actes du magistrat instructeur. Il se détermine par rapport à cette ligne de démarcation fondamentale, dans la procédure criminelle intermédiaire, qui est celle du décret d'accusation, et par rapport au refus des extrêmes. Sa solution est à la fois une solution logique sur le plan procédural, et une solution d'équilibre. Pour Tronchet, seule la sentence contre l'accusé contumace doit être abolie, demeurant au contraire efficaces l'instruction et le récolement des textes. Tronchet récuse également un autre extrême, et c'est celui d'une trop grande faveur faite à l'accusé absent : « II n'est pas possible d'accorder à la représentation de l'accusé l'effet d'anéantir les actes d'instruction qui se sont faits pendant la contumace. Ce serait donner trop d'avantages à l'accusé, au moins suspect $»^{55}$. Pour autant, lors de la

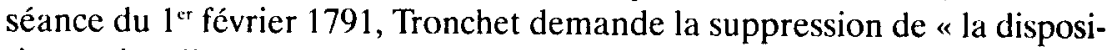
tion qui enlève toute indemnité à l'accusé contumax et de celle qui le condamne, à titre de correction, à un mois de prison ». Duport affirme en effet, dans son rapport sur le projet de décret sur les jurés, que « tout contumax est criminel par cela même qu'il est contumax volontaire ». Duport prêche contre les accusés en fuite un "esprit de sévérité nécessaire " Il propose ainsi, au nom du Comité de Procédure criminelle, de priver l'ac-

(53) Ibid., p. 4, 24 décembre 1789.

(54) Roberto MarTucci, op.cit, chapitre V. p. 178.

(55) Archives parlementaires, p. 4, 24 décembre 1789.

(56) Ibid., l' février 1791, t. XXII, p. 659. 
cusé contumax de l'assistance d'un conseil. Tronchet approuve cette décision sur le fond de l'affaire, mais introduit un amendement disposant que des amis de l'accusé « pourront plaider la légitimité de son excuse ", puis poursuit en désapprouvant la "peine terrible $»^{57}$ que les Anglais réservaient autrefois à ceux qui étaient coupables de défaut de comparution devant les tribunaux, et souligne que « dans un pays libre ", il est essentiel que les hommes “ connaissent l'étendue de la soumission qu'ils doivent à la loi " $^{58}$, mais qu'il faut $y$ « accoutumer les hommes avant de les soumettre à une peine $~^{59}$. Il est approuvé sur ce point par Robespierre, qui refuse la peine prévue pour tout accusé en fuite, et qui s'insurge contre l'article qui porte que « dans le cas d'absolution, l'accusé contumax n'obtiendra aucune indemnité et que le juge le condamnera à garder prison » pendant un mois au plus ; cette disposition méconnaît selon lui « les premiers principes du droit naturel et du bon sens $»^{(x)}$. De même, l'abbé Maury, à la droite de l'assemblée, stigmatise un peu plus tard la « barbarie » des lois anglaises en matière de contumace, et notamment l'absence de révision possible du jugement par contumace ${ }^{n !}$. D'autre part, Tronchet élève des réserves sur la proposition d'article faite par Duport, qui dispose que tous les biens du contumax seront saisis pendant son absence. Il propose que l'on distraie de la " saisie tous les biens propres de la femme et la portion des biens du contumax nécessaire à la subsistance des enfants ${ }^{\text {h2. }}$. Il ne partage pas cependant l'avis de Montlosier, monarchiste obstiné et talentueux, qui refuse toute application de " cette peine odieuse de la confiscation, qui n'était pas connue dans l'Ancien Régime ", pour un accusé qui n'est pas condamné.

C'est le même refus des deux extrêmes qui, quelques mois plus tôt, en décembre 1789 , guide déjà Tronchet et son comité dans leur réponse à la question de savoir quelles seraient les peines à infliger aux citoyens ne voulant pas exercer la fonction de notable-adjoint : « D'un côté, dit-il, la liberté individuelle s'oppose à la contrainte que vous voudriez prononcer; de l'autre la nécessité de maintenir une institution si sage paraît commander cette contrainte. Voilà les deux extrêmes entre lesquels vous avez eu à choisir, Messieurs " $^{6.3}$. En effet la détermination de peines trop sévères aurait pu tomber sous le coup de l'article VIII de la Déclaration des droits

(57) Ibid., $1^{\text {ct }}$ février 1791, t. XXII, p. 660-661 et suiv. Il s'agit vraisemblablement de la peine forte et dure réservée aux accusés qui refusaient le jugement par jury et qui ne voulaient pas faire de déclaration de culpabilité ou de non-culpabilité. Il s'agissait d'un supplice horrible décrit par Victor Hugo dans L'Homme qui Rit. Cf. Adhémar EsMeIN, op. cit., p. 333, note 1.

(58) Ibid.

(59) Ibid.

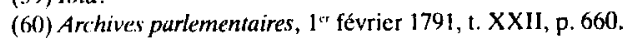

(61) Ibid., p. 662.

(62) Ibid., p. 660

(63) Archives parlementaires, p. 6, 24 décembre 1789. 
de l'homme et du citoyen, qui proscrivait les peines non nécessaires. Tronchet, sans prendre parti, propose au nom du comité un éventail de peines graduées : " La faute peut s'aggraver par degrés, et la peine peutêtre graduée en conséquence ». Il propose donc, au premier refus, la radiation d'un an de la liste des citoyens actifs ${ }^{\text {rt }}$; si celui qui a accepté la mission refuse d'en exercer les fonctions, il préconise une radiation pour deux ans de cette même liste. Enfin, si ce refus s'accompagne de faits de violence qui produisent un scandale et une émeute publique, il préconise la radiation perpétuelle et des poursuites pénales selon la nature des faits. Tronchet en profite pour préciser la nature des notables adjoints : ce sont des surveillants, qui doivent assurer un contrôle remédiant à l'absence de publicité pendant la phase de l'instruction précédant le décret; ce ne sont en aucune manière des juges assimilables aux jurés anglais. La fonction d'adjoint, pour lui, "ne représente pas une étape dans le cursus honorum des citoyens, mais un munus publicum, une charge publique, à remplir dans l'intérêt de la collectivité, et il est, en conséquence, impossible d'y renoncer " ${ }^{\text {ts }}$.

Tronchet démontre le même pragmatisme, probablement hérité de son expérience de praticien, dans le débat sur le caractère écrit de la procédure par jury, qui occupe de nombreuses séances de l'assemblée en janvier 1791 et qui fait partie des débats qui aboutirent à la loi du 16 septembre 1791, qui créa le jury criminel ${ }^{\text {th }}$. La majorité de l'Assemblée constituante désirait l'instauration du jury, "cette institution qui avait grandi avec les libertés anglaises ; les hommes de ce temps avaient le sentiment que cette institution était la marque distinctive des pays libres ${ }^{67}$. Une question se pose cependant : faut-il reprendre le caractère essentiellement oral de la procédure anglaise ou garder le caractère essentiellement écrit de la procédure judiciaire d'Ancien Régime ? Faut-il rejeter le système des preuves de l'Ancien Régime, dit des preuves légales, où la loi écrite dispose des cas de figure précis pour établir la preuve de certains faits ? Faut-il établir le système de la preuve morale, où chaque juré doit décider en conscience de la culpabilité ? Si la plupart des Constituants, y compris Tronchet, s'accordent pour refuser le système des preuves légales, comme nous le verrons par la suite, la discussion est vive quant à la place de l'écrit dans la procédure par jury : est-il possible d'organiser une procédure écrite tout en instaurant le jury?

Dans ce débat, Tronchet se positionne encore comme l'homme de la modération, de la transaction entre deux extrêmes. S'il accepte le système du jury, il préconise son amélioration par l'introduction d'une dose de procédure écrite, héritée de l'ordonnance criminelle de 1670. À l'appui de

(64) Jbid.

(65) Roberto MARTuCC1, op.cit., p. 190.

(66) Jcan-Pierre ROYER, op.cit, p. 285.

(67) Adhémar ESMEIN, op.cit., p. 420. 
ces propositions, il avance des arguments tirés de cas précis se présentant dans la pratique pénale courante. Lintervention de Tronchet pendant la séance du 5 janvier 1791 porte sur la " question de savoir si l'instruction devant le jury de jugement sera écrite ou non ». C'est lors du passage devant le tribunal criminel où siègent douze jurés, le président, le commissaire du roi et l'accusateur public, que la procédure est censée être totalement orale ; ce tribunal intervient après l'enquête du juge de paix et l'inculpation par un jury d'accusation au niveau du district. Les dépositions des témoins ne doivent pas être prises en note, et le scul document écrit reçu par les jurés doit être l'acte d'accusation. Les principaux reproches qu'adresse Tronchet à une procédure totalement orale est qu'elle ne permet pas, en premier lieu, de démontrer un faux témoignage ; en second lieu, elle ne permet pas de susciter de révision du procès en cas d'erreur des jurés ; enfin, l'écrit est nécessaire pour embrasser la complexité de certaines affaires criminelles. Tronchet envisage l'écriture comme un outil de preuve supplémentaire, s'ajoutant aux vertus probatoires de la procédure orale devant le jury, et ne la remettant pas en question.

Sur ces points, il cherche à expliquer les avantages de l'ordonnance criminelle de 1670, mettant à plusieurs reprises ses opposants en difficulté en les confrontant à leur souci d'humanité : «Au moment où vous accusez l'ordonnance de 1670 de barbarie, vous êtes encore plus inhumains qu'elle; vous l'êtes en ôtant à l'accusé presque tous les moyens de se défendre d'un faux témoignage ; vous l'êtes en ôtant la faculté de la révision à l'accusé condamné par une erreur de fait ; vous l'êtes quand vous enlevez à la famille éplorée la faculté de purger sa mémoire ${ }^{\text {bx }}$. Au centre de l'argument de Tronchet sur le jury est l'idée qu'il ne faut pas copier servilement le modèle anglais, en idéalisant la procédure par jury. "Les Anglais, malgré leur extrême attachement à leurs formes, sont bien éloignés de croire à l'infaillibilité du jugement par jury $\aleph^{69}$. En effet, le jury est, pour une bonne partie des Constituants, un hymne à la liberté, en rupture totale avec le système d'Ancien Régime, et une institution véritablement démocratique, symbole même de l'origine populaire de la justice ${ }^{70}$. En cela, l'institution même du jury ne cesse d'être un enjeu politique et symbolique, et Tronchet, fidèle à son attitude transactionnelle, adopte par rapport à cette institution une position de compromis. Il soutient qu'il faut, tout en acceptant la forme du jury, reprendre ce qui dans l'ordonnance criminelle de 1670 pouvait fonctionner, et notamment la trace écrite des confrontations entre témoins.

(68) Archives parlementaires, 5 janvier 1791, t. XXI1, p. 27 et suiv.

(69) lbid.,

(70) Jean-Pierre ROYER, op.cit., p. 284. 
Tronchet est bien l'homme de la transaction entre modernité et tradition ; il se trouve entre deux camps : celui de ceux qui défendent l'oralité intégrale (Duport, Chabroud, Beaumetz, Démeunier) et celui de ceux qui lui sont tout à fait hostiles (Prugnon, Roy, Goupil). Il se trouve à égale distance des imitateurs passionnés des procédures anglaises, comme Duport, et de ceux qui veulent préserver des pans entiers de l'ordonnance criminelle de $1670^{7}$. Sa position, toutefois, ne consiste pas à prôner la transaction par manie du compromis. C'est la conséquence d'une observation attentive de la procédure, qui l'amène à la constatation pragmatique qu'une trace écrite de la procédure devant le jury est nécessaire ; son argumentaire repose en seconde analyse sur des considérations à la fois patriotes et traditionalistes.

Pour expliquer sa position à ses collègues, il choisit à chaque fois des exemples concrets. Ainsi, il expose, que, sans trace écrite des témoignages lors du jugement par jury, il est facile de faire des faux témoignages. Il parle ainsi au nom du condamné victime de faux témoignage : "Quelle preuve aurai-je à opposer aux faux témoins ? Ferai-je entendre le public qui était présent ? Où le retrouverai-je ? Aurai-je le droit de faire entendre le juré qui m'a jugé ? mais il se sera passé six mois, et vous croyez que des jurés, après cet intervalle, oseront se lier assez à leur mémoire pour assurer que c'étaient tels ou tels qui ont fait ces dépositions, plutôt que tels autres ? J'ai donc prouvé inutilement le faux témoignage, et je n'ai pu en obtenir la réparation ${ }^{72}$. C'est la même méthode qu'il emploie pour soutenir que l'absence de trace écrite du procès devant le jury de jugement empêcherait toute révision : "En effet, sans la procédure écrite il m'est impossible de faire connaître quelles étaient les charges qui ont pu me faire condamner, de prouver que tel fait a été ignoré, que tel témoin avait dit cela et était un faussaire " ${ }^{73}$. Là encore, Tronchet prend la parole de celui qui a été accusé injustement, et sa profession d'avocat n'est bien sûr pas étrangère à ce mode de raisonnement, par ailleurs commun dans une élite des professions juridiques marquées par les grandes erreurs judiciaires de la fin du XVIII siècle, des affaires Calas, Sirven ou Montbailli à celles des Cahuzac et Salmon dans les années 1780 ; ensemble, elles avaient démontré la nécessité d'instaurer une procédure de révision facilitée, de rénover le système des preuves, arguments que l'on retrouve dans la discussion sur la procédure écrite dans le jury ${ }^{74}$.

Enfin, Tronchet cherche à montrer que la procédure écrite n'est pas incompatible avec l'institution du jury, et qu'au contraire elle permet de la

(71) Jbid., p. 290 et suiv.

(72) Archives partementaires, 5 janvier 1791, t. XXII, p. 27 et suiv.

(73) $\mathrm{lbid}$.

(74) Benoît Garnot, Questions de justice, 1667-1789, Paris, Belin, 2006, p. 118 et suiv. 
renforcer. Si Tronchet accepte la théorie de la conviction morale, celle par qui le juré condamne au nom de son "intime conviction ", et s'il réfute la théorie des preuves légales ${ }^{75}$, par laquelle, sous l'Ancien Régime, une partie de la doctrine prétendait déterminer par la loi l'ensemble des critères permettant de condamner un accusé, il considère cependant que l'institution même du jury serait renforcée par l'ajout d'un autre mode de preuve, cette fois écrite : « Le fait écrit rappellera au juré bien plus facilement les détails moraux de la discussion, que les détails moraux ne lui rappelleront les détails positifs, dont sa mémoire se trouvera surchargée, si les circonstances sont un peu compliquées $"{ }^{76}$. Il conclut par une formule qui frappa les députés de l'assemblée et qui explique pourquoi son discours fit « tant de sensation $"{ }^{77}$ sur la Constituante : "En un mot avec l'écriture je réunis deux avantages ; sans elle vous n'en avez qu'un, et toute la question entre vous et moi se réduit à décider si un vaut mieux que deux " $"$. Tronchet partage ici la position d'autres avocats et Constituants, comme Rey et Robespierre. Robespierre et Tronchet mettaient en effet en garde leurs collègues contre les dangers d'une procédure où l'on laissait toute latitude à l'intime conviction du juré pour décider. Si Robespierre tenait pour une combinaison des preuves légales et de l'intime conviction, Tronchet, lui, affirmait son refus du système des preuves légales d'Ancien Régime, et préconisait l'écrit comme mode de preuve supplémentaire.

La position de Tronchet, si elle ne s'imposa pas tout de suite, fut adoptée dans les réformes suivantes de la procédure criminclle, notamment dans le Code du 3 brumaire an IV, qui introduisit une part d'écrit dans la procédure devant le jury ${ }^{79}$. Elle est marquée également par le formalisme juridique, qui réapparaîtra lors du procès du roi, où Tronchet mettra en avant des considérations de procédure. Tronchet appartient à cette école de pensée juridique qui valorise le formalisme, et pense, tel Ihering, que, « ennemie juré de l'arbitraire, la forme est la jumelle de la liberté " ${ }^{8 \prime \prime}$. Ce formalisme juridique, en temps de révolution, est-il un oubli de la politique au profit du droit ? Ou est-ce, en soi, une politique, que de refuser les considérations purement politiques pour se cantonner au formalisme juridique ? Cet attachement au formalisme est-il un réflexe professionnel?

(75) « Jamais législateur n'a été assez absurde pour imaginer qu'il pût prescrire à des juges des règles infaillibles, et généralement applicables pour prononcer sur des faits et d'après les dispositions des témoins $»$.

(76) Archives parlementaires, 5 janvier 1791, t. XXII, pages 27 et suiv.

(77) lbid.

(78) Ibid.

(79) Adhémar Esmeın, op.cit, p. 434 et suiv. Larticle 365 du code de Brumaire dispose que " les procès verbaux des témoignages, et les notes écrites prises pendant les interrogatoires [...] peuvent être lus aux jurés pendant le déroulement du procès" "

(80) Rudolph von Lhering, Le Combat pour le Droit, Paris, Durand et Pedone, 1875. 
Dans le discours que Tronchet prononce sur le caractère écrit de la procédure devant le jury, apparaît en effet son ethos d'avocat, c'est-à-dire sa propension naturelle à envisager les cas de la pratique et à se mettre du côté de l'accusé. Tronchet glorifie l'idéal du bon avocat, fidèle aux règles de sa profession, qui exigent le "désintéressement ${ }^{81}$, mais aussi le courage devant les pouvoirs et la disposition à la lutte pour la défense du faible et de l'innocent. Ainsi affirme-t-il, à propos des notables-adjoints qui pourraient refuser leur mission : "Défendre un accusé est une fonction si honorable pour l'humanité ! Comment pourrait-elle être refusée par un citoyen quelconque ? Quel prétexte pourrait légitimer ce refus ? Ce n'est pas la gravité du crime ; l'accusé n'est peut-être pas coupable. Plus l'accusation est grave, plus il a le droit d'être défendu, s'il est innocent $\$^{{ }^{2}}$.

La tirade prend un son singulier dans la bouche de celui qui sera l'avocat de Louis XVI. Pour Martucci, la fonction de conseil de l'accusé, dans le discours de Tronchet, est assimilable à celle d'adjoint, devenant elle aussi un munus publicum, une charge publique, de laquelle il est impossible de se détacher, sauf motif d'exemption ${ }^{83}$. Le système de pensée de Tronchet est très marqué, comme celui de bien des révolutionnaires, par l'idéal de la cité antique et de la Rome républicaine où, dans une vision idéalisée, la vertu égalitaire allait de pair avec le désintéressement civique. Cette apologie du désintéressement n'empêchera pas Tronchet de réaliser une ascension sociale remarquable pendant la période révolutionnaire et impériale, passant d'une frange modeste des professions juridiques, celle des avocats, au sommet de l'État et au coeur du pouvoir, en l'occurrence le Sénat conservateur ; cette ascension vers le pouvoir va de pair avec un enrichissement qui apparaît dans son testament ${ }^{84}$.

Le bon avocat ou le bon juge doit également, pour Tronchet, être fidèle à la tradition d'étude et de science juridique des hommes de loi. Le personnage est attaché à la figure du jurisconsulte ${ }^{\mathrm{ks}}$ cultivé, étudiant les précédents et la tradition, cultivant ses « lumières personnelles ${ }^{\star \mathrm{kh}}$. Plaidant pour que les tribunaux d'appel soient sédentaires, Tronchet explique par exemple qu'il " est des cas nouveaux où le juge le plus instruit est obligé de consulter des livres. Mais qu'ai-je dit, les livres ? on prétend qu'il faut les brûler, qu'il faut livrer au feu tous ces in-folio qui garnissent nos bibliothèques. Heureusement pour nos libraires, que l'on a pas dit qu'il fallait livrer aux flammes les livres d'histoire, de science et de littérature. Quant à

(81) Hervé LeUwERS, L'Invention du harreau français, 1660-1830, La construction nationale d'un groupe professionnel, Paris, Éditions EHESS, 2006. p. 190 et suiv.

(82) Archives parlementaires, 24 décembre 1789 , I. XI, p. 3 et suiv.

(83) Roberto MARTUCCI, op.cit., chapitre V, p. 190.

(84) AN, minutier central, étude LXXXVI, liasse 941 (janvier- avril 1806).

(85) Hervé LEUWERS, L'invention du barreau français..., op. cit., p. 173 et suiv.

(86) Archives parlementaires, 3 mai 1790, t. XV, p. 362 et suiv. 
ceux que vous avez rendus inutiles, j'en ferai bien volontiers le sacrifice ; mais je demande grâce pour quelques autres, car je leur dois le peu que je vaux $"{ }^{87}$. Tronchet attaque ici avec ironie la doctrine de la table rase qui a pu inspirer certains révolutionnaires dans leur volonté de rompre avec l'Ancien Régime. C'est aussi une considération pragmatique où Tronchet se met à la place du justiciable : « Je n'aurai jamais de confiance en un juge qui viendra décider de ma fortune en portant toute sa science en croupe sur son cheval ${ }^{88}$.

À observer les positions de Tronchet dans le débat sur la réforme judiciaire, c'est un réformateur attaché aux valeurs d'équilibre inséparables de la pratique de sa profession d'avocat qui apparait. S'écartant des traditionalistes attachés à l'ordonnance de 1670 , mais aussi de ceux qui croyaient dans l'infaillibilité du modèle anglais, il se fit alors le défenseur d'une transaction à la française. Plus largement, il apparaît à la fois comme refusant la « barbarie » des supplices et des mesures extrêmes - barbarie qu'il refuse d'associer systématiquement à l'Ancien Régime -, et rejetant l'idéalisation des modèles étrangers. Si, sur le jury, il se range aux côtés de magistrats conservateurs comme Mougin, ou de monarchistes comme Maury, ses positions sont également partagées par Robespierre, qui, de façon surprenante, soutient le maintien de la théorie des preuves légales par écrit. On pourrait faire de mêmes remarques concernant l'histoire des réformes du droit civil où, comme la majeure partie de l'Assemblée, il accepte des transformations immédiates tout en considérant que la codification peut-être repoussée à plus tard.

Il est bien difficile de " classer » Tronchet. En premier lieu, les discussions techniques portant sur la réforme de la procédure criminelle amènent les protagonistes à des positions juridiques ne correspondant pas à leur positionnement habituel sur l'échiquier politique, comme c'est le cas pour Robespierre... Peut-être parce que l'expérience professionnelle de ces avocats leur a donné des lumières particulières sur ces problèmes techniques. En second lieu, il faut souligner la constance de Tronchet dans son itinéraire de pensée : il semble, à chaque fois, plus attaché à la logique des principes et des pratiques juridiques, qu'aux logiques politiques, qu'il semble mettre à l'écart. À cet égard, c'est peut-être un des exemples d'une pensée qui fit passer le formalisme juridique avant le politique, et qui fut d'abord vaincue dans la première partie de la Révolution, pour triompher ensuite dans la remise en ordre de Bonaparte. Cela dit, la spécificité des positions de Tronchet ne peut s'expliquer par sa seule origine professionnelle ; 
elles s'expliquent aussi - et peut-être d'abord - par une certaine vision du droit, qui plonge ses racines dans son expérience et ses convictions, qui l'amènent à une théorie du respect des formes et de la juste mesure comme corollaire de la nécessaire humanité de l'organisation judiciaire.

Philippe TESSIER

Université Lille 3/IRHiS

BP 60149

59653 Villeneuve-d'Ascq

tessierphl@free.fr 